\title{
TRANSLOCAÇÃO DOS HERBICIDAS GLYPHOSATE E IMAZAMOX EM PLANTAS DE AGUAPÉ
}

\author{
C.F. de Campos, H.S. Vitorino, G.S.F. de Souza, D.C. de Santana, D. Martins
}

Universidade Estadual de São Paulo, Faculdade de Ciências Agronômicas, Fazenda Lageado, CP 237, CEP 18610-307, Botucatu, SP, Brasil. E-mail: caio.agro@hotmail.com

\section{RESUMO}

\begin{abstract}
Entre as diversas plantas daninhas encontradas em ambientes aquáticos, a Eichhornia crassipes é uma das principais, sendo espécie indesejável para muitas atividades. O objetivo deste trabalho foi avaliar a translocação de glyphosate e imazamox em plantas de E. crassipes. Foram estudados oito intervalos de tempo para o corte das folhas que receberam os herbicidas: 2, 4, 6, 8, 12 e 24 horas após a aplicação (HAA) dos produtos, além de um tratamento sem corte das plantas (testemunha). Utilizaram-se glyphosate na dose de 2.160 g e.a. ha-1 (produto comercial - Rodeo) + 0,5\% v v vo $^{-1}$. espalhante adesivo aterbane e imazamox na dose de 290,4 g i.a. ha-1 (produto comercial - Clearcast). Os tratamentos foram instalados em um delineamento experimental inteiramente casualizado com quatro repetições. O glyphosate apresentou um controle insatisfatório quando realizado os cortes das folhas em todos os períodos avaliados. O herbicida imazamox não proporcionou controle em até $12 \mathrm{HAA}$, enquanto que a partir de $24 \mathrm{HAA}$ do herbicida o controle foi eficiente. Não houve uma grande mobilidade da molécula de glyphosate nas plantas de aguapé, sendo necessário um período superior a 24 horas para uma translocação satisfatória. Já para o imazamox um tempo de mínimo de 24 horas após a aplicação do herbicida foi suficiente para que ocorresse a translocação e um posterior controle.
\end{abstract}

PALAVRAS-CHAVE: Planta aquática, planta daninha, tecnologia de aplicação, Eichhornia crassipes.

\section{ABSTRACT}

GLYPHOSATE AND IMAZAMOX TRANSLOCATION IN WATER HYACINTH PLANTS. Eichhornia crassipes is one of the main weeds found in aquatic environments, being undesirable for many activities. The aim of this study was to evaluate the translocation of glyphosate and imazamox in E. crassipes. Eight intervals were studied for cutting leaves that received herbicides: 2, 4, 6, 8, 12 and 24 hours after application (HAA), and a treatment with no cutting (untreated). The glyphosate dose was 2,160 g a.e. ha-1 (commercial product - Rodeo) $+0.5 \% \mathrm{v} \mathrm{v}^{-1}$ Aterbane adhesive spreader and imazamox at $290.4 \mathrm{~g}$ i.a. ha ${ }^{-1}$ (commercial product - Clearcast). The treatments were installed in a completely randomized design with four replications. Glyphosate showed a bad control for all the periods of leaf cutting. The imazamox did not provide control within 12 HAA, while from 24 HAA onward the control was effective. There was not a great mobility of the glyphosate molecule in water hyacinth plants, a period above 24 hours being needed for a satisfactory translocation. For imazamox at least 24 hours were needed after herbicide application for the translocation to occur along with subsequent control.

KEY WORDS: Aquatic plant, weed, spraying technology, Eichhornia crassipes.

\section{INTRODUÇÃO}

Espécies de plantas aquáticas flutuantes possuem em geral grande plasticidade fenotípica, reprodução vegetativa intensa e elevada taxa de crescimento (Rubim; Camargo, 2001; Henry-Silva et al., 2008). Podem ser encontradas dentro dos mais diversos ambientes aquáticos, por utilizaram-se de diferentes mecanismos de adaptação (MARTins et al., 2009), a exemplo do aguapé.
A espécie é uma planta agressiva e apresenta enorme potencial de reprodução, o que confere a capacidade em aumentar em $15 \%$ ao dia sua área de cobertura, que pode ser dobrada a cada semana (HOYER et al., 1996). Esse caráter invasor deve-se à sua alta capacidade de multiplicação vegetativa, fazendo com que grandes extensões sejam ocupadas rapidamente (GuITIÉRREZ et al., 1996).

Sua proliferação de forma desorganizada foi intensificada devido ao processo de autodepura- 
ção realizado pelos organismos ao transformarem o material biodegradável, oriundo da descarga de resíduos, em nutrientes. $\mathrm{O}$ crescimento intenso das espécies aquáticas, o que inclui aguapé, cobre a superfície livre dos corpos d'água, e dificulta o acesso e sua utilização (Neves et al., 2002).

Ocontrolequímicoéum método promissore pode ser feito de forma segura como glyphosate (MARTINS et al., 2002; Neves et al., 2002; CARVALHO et al., 2005) e imazamox (WERSAL; MADSEN, 2007).

O herbicida glyphosate é um inibidor enolpiruvil shikimato-3-fosfato sintase (EPSPS) e sua ação sistêmica, associada à baixa toxicidade para pássaros, peixes e bactérias, contribui para estudos que exploram seu uso em ambientes aquáticos, por ser facilmente translocado e provocar a morte do sistema radicular e de estruturas reprodutivas (KRUSE et al., 2000).

O glyphosate movimenta-se no floema seguindo a rota dos produtos da fotossíntese, das folhas fotossinteticamente ativas (fonte) em direção às partes das plantas em crescimento (dreno), dessa forma, a absorção, a translocação e o metabolismo do herbicida podem afetar a sensibilidade de uma planta, pois este precisa atingir o sítio de ação numa concentração adequada (HETHERINGTON et al., 1999).

Outra opção é o herbicida imazamox que pertence ao grupo químico das imidazolinonas, tem o seu local de ação na enzima acetolactato sintase (ALS). Como o sistema enzimático é exclusivo das plantas e a toxicidade do produto é baixa para peixes e invertebrados aquáticos, o herbicida também tem potencial de uso no manejo das plantas aquáticas (Shaner, 1991; MAlliPudi, 1991). Entretanto, como o imazamox é rapidamente absorvido pelas folhas e translocado na planta via floema e o xilema (SHANER, 1991; MALLIPUDI, 1991; WeED, 2002), acredita-se ser mais difícil a remoção do herbicida da planta para os cursos d'água.

Dessa forma, o estudo teve como objetivo avaliar o tempo necessário à translocação dos herbicidas glyphosate e imazamox para um efetivo controle das plantas de Eichhornia crassipes.

\section{MATERIAL E MÉTODOS}

O estudo com E. crassipes (aguapé) foi conduzido no Núcleo de Pesquisas Avançadas em Matologia (NUPAM), da FCA/UNESP, campus de Botucatu, $\mathrm{SP}$, em condições de casa de vegetação a $26^{\circ} \mathrm{C}$.

As plantas aquáticas foram coletadas na várzea da Fazenda Edgardia, pertencente à Universidade e mantidas individualmente em vasos plásticos de 15 $\times 15 \times 15$ cm, com 2,5 L de água. Respeitou-se um período de duas semanas para aclimatação das plantas ao novo ambiente antes da ocasião da aplicação do herbicida. Diariamente foi realizada a manutenção da água dos vasos para manter o volume inicial.

A área de ocupação média de 20 plantas de aguapé $\mathrm{m}^{-2}$ foi estimada para estabelecer a calda de pulverização necessária para um bom recobrimento das plantas e a concentração do produto na dose comercial. Dessa forma, fez-se a simulação através de micropipeta de um tamanho de gota que não escorresse no momento da aplicação, assim, encontrou-se um volume de gota de $0,03 \mathrm{~mL}$. A gota foi depositada com uma micropipeta sobre a face adaxial de uma folha a qual foi cortada de acordo com os intervalos de tempo de cada tratamento estudado.

Utilizou-se o delineamento inteiramente casualizado com os tratamentos em quatro repetições. Os tratamentos foram constituídos por intervalos de tempo: 2, 4, 6, 8, 12 e 24 horas após a aplicação dos herbicidas, além da testemunha sem cortee aplicação.

Aplicaram-se os herbicidas glyphosate $(2.160 \mathrm{~g}$ e.a. ha ${ }^{-1}$ - marca comercial Rodeo) acrescido $0,5 \% \mathrm{v}$ $\mathrm{v}^{-1}$ de espalhante adesivo aterbane e imazamox (290 g i.a. ha ${ }^{-1}$ - marca comercial Clearcast).

As avaliações visuais de controle foram realizadas aos 7, 14, 21, 28 e 35 dias após a aplicação (DAA) dos tratamentos, através da escala de percentual de notas, na qual 0 (zero) correspondeu a nenhuma injúria e 100 (cem) a morte das plantas, (SOCIEDADE, 1995). Os parâmetros utilizados para estabelecimento das notas foram inibição do crescimento, capacidade de rebrota, além da quantidade e uniformidade das injúrias das plantas. Aofinal da avaliação aos $35 \mathrm{DAA}$ foi determinada a massa seca das plantas. As 2, 4, $6,8,12$ e 24 horas após a aplicação dos herbicidas (HAA), também tomou-se a folha que em seguida era descartada.

Os resultados foram submetidos à análise de variância pelo Teste " $F$ " e as médias dos tratamentos foram comparadas pelo teste de Tukey a $5 \%$.

\section{RESULTADOS E DISCUSSÃO}

Os resultados observados aos 7 dias após a aplicação (DAA) demonstraram que não houve injúrias nas plantas de aguapé quando aplicado o glyphosate e realizado o corte da folha que recebeu o herbicida em até 4 horas após a aplicação (HAA) do herbicida. Quando não houve a realização do corte da folha foi atribuída a maior nota de fitotoxicidade para as plantas de aguapé (Tabela 1).

Aos 14 DAA, houve um grande incremento nas injúrias apresentadas pelas plantas de aguapé e os menores valores de fitotoxicidade foram observados quando o corte da folha foi realizado até $8 \mathrm{HAA}$, assim, de acordo com HEss; FALK (1990), antes de apresentar ação fitotóxica, o herbicida deve ser absorvido via apoplasto e/ou simplasto e alcançar o seu sítio 
de ação, geralmente localizado no interior de uma organela, o que provavelmente foi dificultado pelo curto intervalo de tempo. Porém, no tratamento em que não foi realizado o corte (testemunha com aplicação de herbicida), já houve um controle acima de $80 \%$ considerado bom de acordo com a SOCIEDADE... (1995).

Houve também um incremento nos níveis de controle, aos 21 DAA, porém, os menores controles ainda estavam presentes quando o corte foi realizado até 8 HAA do herbicida, possivelmente pelo tempo de translocação menor, dessa forma, destaca-se o tratamento sem a realização do corte com um controle de $92,5 \%$. SiLva (1996) observou que há necessidade de um período sem chuva superior a 16 horas para que houvesse o máximo de efeito do glyphosate sobre Brachiaria decumbens.

Aos 28DAA, houveumleveincrementonos níveis de controle das plantas de aguapé mantendo o maior efeito da fitotoxicidade no tratamento testemunha sem corte da folha que recebeu o herbicida, o que sugere um maior tempo de translocação do herbicida. No final do estudo, aos 35 DAA, a avaliação visual manteve-se praticamente inalterada e, para o tratamento testemunha, o controle proporcionado pelo herbicida glyphosate foi considerado muito bom, com valor igual a 96\% (SOCIEDADE..., 1995).

Dessa forma, pode-seobservar quehánecessidade de mais do que 24 horas para que a translocação de uma quantidade suficiente do herbicida glyphosate para proporcionar um controle eficiente das plantas de aguapé. Portanto, de acordo com WerLANG et al. (2003), que, após atingir a superfíciefoliar, o herbicida está sujeito a vários destinos: escorrer, ser lavado pela ocorrência de chuva, secar e formar substância amorfa, cristalizar após a evaporação do solvente ou, ainda, penetrar na cutícula e permanecer retido nela, não sendo translocado, tendo menor absorção e eficácia.

Em estudo realizado por MARTins et al. (2009) foi observado não haver uma grande mobilidade

Tabela 1 - Porcentagem de controle de E. crassipes, em função dos períodos de corte das folhas após aplicação de glyphosate $\left(2.160 \mathrm{~g} \mathrm{ha}^{-1}\right)$. Botucatu, SP, 2011.

\begin{tabular}{lccccc}
\hline \multirow{2}{*}{ Períodos } & \multicolumn{5}{c}{ Dias após a aplicação } \\
\cline { 2 - 6 } & 7 & 14 & 21 & 28 & 35 \\
\hline 2 horas & $0,00 \mathrm{e}$ & $9,25 \mathrm{c}$ & $20,50 \mathrm{c}$ & $23,25 \mathrm{~d}$ & $22,50 \mathrm{c}$ \\
4 horas & $0,00 \mathrm{e}$ & $15,00 \mathrm{c}$ & $20,75 \mathrm{c}$ & $27,75 \mathrm{~d}$ & $27,00 \mathrm{c}$ \\
6 horas & $4,25 \mathrm{de}$ & $18,50 \mathrm{c}$ & $26,00 \mathrm{c}$ & $32,50 \mathrm{~d}$ & $33,75 \mathrm{c}$ \\
8 horas & $7,75 \mathrm{cde}$ & $23,50 \mathrm{c}$ & $29,25 \mathrm{c}$ & $33,75 \mathrm{~cd}$ & $35,00 \mathrm{c}$ \\
12 horas & $12,50 \mathrm{bc}$ & $49,50 \mathrm{~b}$ & $52,00 \mathrm{~b}$ & $50,75 \mathrm{bc}$ & $57,50 \mathrm{~b}$ \\
24 horas & $16,25 \mathrm{~b}$ & $54,00 \mathrm{~b}$ & $54,25 \mathrm{~b}$ & $63,75 \mathrm{~b}$ & $70,00 \mathrm{~b}$ \\
Sem corte & $27,75 \mathrm{a}$ & $80,25 \mathrm{a}$ & $92,50 \mathrm{a}$ & $97,25 \mathrm{a}$ & $96,00 \mathrm{a}$ \\
\hline $\mathrm{F}_{\text {TRATAMENTO }}$ & $18,05^{* *}$ & $42,58^{* *}$ & $68,85^{* *}$ & $49,65^{* *}$ & $46,4^{* *}$ \\
CV (\%) & 48,0 & 22,4 & 15,0 & 15,9 & 16,2 \\
d.m.s. & 10,81 & 18,28 & 14,54 & 17,14 & 18,22 \\
\hline
\end{tabular}

**significativo a $1 \%$ de probabilidade.

Tabela 2 - Porcentagem de controle de E. crassipes, em função dos períodos de corte das folhas após aplicação de imazamox (290,4 $\left.\mathrm{g} \mathrm{ha}^{-1}\right)$. Botucatu, SP, 2011.

\begin{tabular}{lccccc}
\hline \multirow{2}{*}{ Períodos } & \multicolumn{4}{c}{ Dias após a aplicação } \\
\cline { 2 - 6 } & 7 & 14 & 21 & 28 & 35 \\
\hline 2 horas & $0,00 \mathrm{~b}$ & $13,00 \mathrm{~b}$ & $15,00 \mathrm{c}$ & $17,50 \mathrm{~d}$ & $7,50 \mathrm{~d}$ \\
4 horas & $0,00 \mathrm{~b}$ & $14,00 \mathrm{~b}$ & $23,25 \mathrm{bc}$ & $25,75 \mathrm{~cd}$ & $23,75 \mathrm{c}$ \\
6 horas & $0,00 \mathrm{~b}$ & $18,25 \mathrm{~b}$ & $33,00 \mathrm{~b}$ & $35,00 \mathrm{bc}$ & $46,25 \mathrm{~b}$ \\
8 horas & $0,00 \mathrm{~b}$ & $22,50 \mathrm{~b}$ & $34,25 \mathrm{~b}$ & $39,25 \mathrm{~b}$ & $48,75 \mathrm{~b}$ \\
12 horas & $3,00 \mathrm{~b}$ & $24,25 \mathrm{~b}$ & $35,50 \mathrm{~b}$ & $38,75 \mathrm{bc}$ & $52,50 \mathrm{~b}$ \\
24 horas & $13,75 \mathrm{a}$ & $56,25 \mathrm{a}$ & $80,75 \mathrm{a}$ & $83,25 \mathrm{a}$ & $87,50 \mathrm{a}$ \\
Sem corte & $10,75 \mathrm{a}$ & $58,00 \mathrm{a}$ & $88,75 \mathrm{a}$ & $94,25 \mathrm{a}$ & $96,25 \mathrm{a}$ \\
\hline $\mathrm{F}_{\text {TRATAMENTO }}$ & $41,41^{* *}$ & $33,30^{* *}$ & $82,26^{* *}$ & $105,34^{* *}$ & $120,80^{* *}$ \\
CV (\%) & 46,3 & 22,7 & 14,2 & 12,0 & 11,2 \\
d.m.s. & 4,18 & 15,41 & 14,51 & 13,11 & 13,27 \\
\hline
\end{tabular}

**significativo a $1 \%$ de probabilidade. 
da molécula do glyphosate em até 4 horas em plantas de aguapé, o que corrobora os resultados encontrados neste estudo. Contudo, MonQuero et al. (2004) observaram em plantas de Amaranthus hybridus, Ipomoea grandifolia e Commelina benghalensis que apenas 2,$85 ; 2,75$ e $0,96 \%$, respectivamente, do herbicida aplicado foram encontrados fora da folha que recebeu o glyphosate 12 horas após a sua aplicação, o que resultou em um baixo efeito no controle dessas plantas.

Quanto ao efeito do herbicida imazamox em plantas de aguapé (Tabela 2), foram observadas aos 7 DAA apenas leves injúrias nas plantas de aguapé, porém, os melhores resultados ocorreram quando houve um corte 24 horas após a aplicação e a não realização do corte (testemunha), sendo que não foram verificadas injúrias nas plantas nas quais foram submetidas ao corte da folha que recebeu o herbicida até 8 horas após a sua aplicação.

Aos 14DAA houve um acentuado incremento no controle, porém, ainda insatisfatórios em todos os tratamentos, sendo que dentre os tratamentos testados, os menores controles foram registrados quando da realização do corte até 12 horas após a aplicação do herbicida imazamox. Na avaliação visual realizado aos 21 DAA, um controle satisfatório (acima de $80 \%$ ) foi observado quando houve o corte 24 horas após a aplicação e quando não o realizado (testemunha). Diferente do herbicida glyphosate, o imazamox é rapidamente absorvido pelas folhas e translocado através da planta via floema e xilema (WEED, 2002), fato esse que proporcionou um controle superior a $80 \%$ das plantas daninhas de aguapé.

Aos 28 DAA, não houve controle quando se realizou o corte em até 12 HAA. Após 24 HAA foi observado um controle acima de $80 \%$ das plantas de aguapé e de acima de $94 \%$ quando não houve a realização do corte da folha (testemunha). CAMPOS et al. (2012) observaram um controle de 98,25\% do imazamox sobre plantas de aguapé (E. crassipes) aos 28 DAA.

Na avaliação visual aos 35 DAA foi registrada uma recuperação das plantas de aguapé submetidas ao corte em até 2 HAA. As 12 HAA não houve controle das plantas de aguapé, tendo como valor máximo de controle $52,5 \%$.Quando realizado o corte 24 horas após a aplicação e sem o corte (testemunha), o herbicida imazamox proporcionou um controle adequado segundo a SOCIEDADE (1995), considerados bom e muito bom.

Pode-se considerar que 24 horas foi um período hábil para que ocorresse a translocação de uma quantidade suficiente para o controle das plantas de aguapé quando comparado a uma situação em que a planta tenha um tempo indeterminado para a realização da translocação. A não ocorrência da morte das plantas que não tiveram a folha aplicada cortada, mas o alto grau de injúrias indicou haver uma grande translocação do herbicida, no entanto, insuficiente para a morte da planta.

Contudo, o resultado obtido com um corte 24 HAA do herbicida imazamox mostra-se a mais próxima da condição máxima de translocação. O mesmo foi observado por Pester et al. (2001) que encontraram moléculas de imazamox nas raízes de Aegilops cylindrica a partir de 12 horas após a aplicação, indicando uma grande movimentação da molécula dentro da planta.

Ao analisar o acúmulo de massa seca das plantas de aguapé submetidas à aplicação de glyphosate

Tabela 3 - Massa seca de diferentes partes de E. crassipes, em função dos períodos de corte das folhas após aplicação de glyphosate (2.160 $\left.\mathrm{g} \mathrm{ha}^{-1}\right)$. Botucatu, SP, 2011.

\begin{tabular}{lccc}
\hline \multirow{2}{*}{ Períodos } & \multicolumn{3}{c}{ Massa seca $(\mathrm{g})$} \\
\cline { 2 - 4 } & Raiz & Parte aérea & Total \\
\hline testemunha & $2,64(2,10)$ & $4,99(2,72)$ & $7,63(3,24)$ \\
2 horas & $2,52(2,03)$ & $4,49(2,57)$ & $7,01(3,08)$ \\
4 horas & $2,98(2,16)$ & $4,61(2,64)$ & $7,59(3,23)$ \\
6 horas & $2,10(1,95)$ & $3,37(2,32)$ & $5,47(2,83)$ \\
8 horas & $2,73(2,14)$ & $3,06(2,24)$ & $5,79(2,89)$ \\
12 horas & $2,43(2,02)$ & $2,78(2,11)$ & $5,21(2,72)$ \\
24 horas & $2,32(2,02)$ & $3,03(2,23)$ & $5,35(2,81)$ \\
Sem corte & $2,25(1,97)$ & $2,49(2,04)$ & $4,74(2,63)$ \\
\hline F & $0,19^{\text {ns }}$ & $2,12^{\text {ns }}$ & $0,95^{\text {ns }}$ \\
CV (\%) & 17,6 & 14,7 & 16,1 \\
d.m.s. & 0,84 & 0,81 & 1,10 \\
\hline
\end{tabular}

${ }^{\text {nsnão significativo. Dados entre parênteses são resultados }}$ da transformação: $\sqrt[2]{x}+0,5$.

Tabela 4 - Massa seca de diferentes partes de E. crassipes, em função dos períodos de corte das folhas após aplicação de imazamox (290,4 $\left.\mathrm{g} \mathrm{ha}^{-1}\right)$. Botucatu, SP, 2011.

\begin{tabular}{lccc}
\hline \multirow{2}{*}{ Períodos } & \multicolumn{3}{c}{ Massa seca $(\mathrm{g})$} \\
\cline { 2 - 4 } & Raiz & Parte aérea & Total \\
\hline testemunha & $3,03(2,23)$ & $5,45(2,83) \mathrm{a}$ & $8,48(3,40) \mathrm{a}$ \\
2 horas & $3,12(2,24)$ & $5,50(2,84) \mathrm{a}$ & $8,62(3,42) \mathrm{a}$ \\
4 horas & $2,74(2,10)$ & $4,50(2,61) \mathrm{ab}$ & $7,24(3,17) \mathrm{ab}$ \\
6 horas & $2,14(1,93)$ & $3,18(2,22) \mathrm{abc}$ & $5,32(2,74) \mathrm{ab}$ \\
8 horas & $2,18(1,98)$ & $2,44(2,06) \mathrm{bc}$ & $4,62(2,65) \mathrm{ab}$ \\
12 horas & $2,71(2,14)$ & $2,91(2,20) \mathrm{abc}$ & $5,62(2,87) \mathrm{ab}$ \\
24 horas & $2,39(2,03)$ & $2,43(2,04) \mathrm{bc}$ & $4,82(2,68) \mathrm{ab}$ \\
Sem corte & $1,56(1,69)$ & $2,08(1,91) \mathrm{c}$ & $3,65(2,35) \mathrm{b}$ \\
\hline F & $1,13^{\text {ns }}$ & $6,31^{* *}$ & $4,27^{* *}$ \\
TRATAmENTO & 15,7 & 12,6 & 12,8 \\
d.m.s. & 0,75 & 0,69 & 0,87 \\
\hline
\end{tabular}

**significativo a 1\% de probabilidade; ns não significativo. Dados entre parênteses são resultados da transformação: $\sqrt[2]{x}+0,5$. 
observou-se que, quando comparado à raiz e à parte aérea das plantas, não houve diferença entre os tratamentos e, consequentemente, a soma das suas partes, porém, em termos biológicos pode-se notar a existência de uma redução gradual do acúmulo de biomassa quando as plantas apresentaram maiores injúrias.

As plantas tratadas com imazamox (Tabela 4) não apresentaram diferenças para os diferentes períodos de corte quando analisada a massa seca das raízes, no entanto, para a parte aérea foi observado um menor acúmulo de biomassa nas plantas que foram submetidas ao corte 8,24 HAA e quando da não realização do corte da folha tratada. Quando analisado o efeito do herbicida imazamox sobre o acúmulo de massa seca em toda a planta, verificouse um menor acúmulo no tratamento sem corte da folha que recebeu o produto, corroborando com o controle apresentado pelo herbicida aos 35 DAA.

\section{CONCLUSÃO}

Houve baixa mobilidade da molécula de glyphosate nas plantas de aguapé, sendo necessário um período superior a 24 horas para que a translocação ocorra com eficiência. Oimazamox proporcionou um controle eficiente no tempo mínimo de 24 HAA do herbicida o qual foi suficiente para que ocorresse a translocação e controle.

\section{REFERÊNCIA}

CAMPOS, C.F.; SOUZA, G.S.F. de; MARTINS, D.; PEREIRA, M.R.R.; BAGATTA, M.V.B. Influência da chuva após a aplicação de imazamox sobre o controle de plantas daninhas aquáticas. BioScience Journal, v.28, n.3, p.413-419, 2012

CARVALHO, F.T.; VELINI, E.D.; NEGRISOLI, E.; ROSSI, C.V.S. Eficácia do carfentrazone-ethyl no controle de plantas aquáticas latifoliadas em caixas-d'água. Planta Daninha, v.23, n.2, p.305-310, 2005.

GUTIÉRREZ, E.; HUERTO, R.; SALDAFIA, P.; ARREGUIN, F. Strategies for waterhyacinth (Eichhornia crassipes) control in Mexico. Hydrobiologia, v.340, n.1, p.181-185, 1996.

HENRY-SILVA, G.G.; PEZZATO, M.M.; CAMARGO, A.F.M. Growth of free-floating aquatic macrophytes in different concentrations of nutrients. Hydrobiologia. v.610, n.1, p.153-160, 2008.

HESS, F.D.; FALK, R.H. Herbicide deposition on the leaf surface. Weed Science, v.38, n.3, p.280-288, 1990. HETHERINGTON, P.; REYNOLDS, T.L.; MARSHALL, G.; KIRKWOOD, R.C. The absorption, translocation and distribution of the herbicide glyphosate in maize expressing the CP-4 transgene. Journal of Experimental Botany, v.50, n.339, p.1567-1576, 1999.

HOLM, L.G.; WELDON, L.W.; BLACKBURN R.D. Aquatic seeds. Science, v.166, n.2, p.699-709, 1969.

HOYER, M.V.; CANFIELD JUNIOR, D.E.; HORSBURGH, C.A.; BROWN, K. Florida freshwater plants: a handbook of commom aquatic plants in Florida lakes. Gainesville: University of Florida, Institute of Food and Agriculture Sciences, 1996. 264p.

KRUSE, D.N.; TREZZI, M.M.; VIDAL, R.A. Herbicidas inibidores da EPSPS: revisão de literatura. Revista Brasileira de Herbicidas, v.1, n.2, p.139-146, 2000.

LAREO, L. Crecimiento de jacinto de água (Eichhornia crassipes (Mart) Solms Laubach). Archivos Latinoamericanos de Nutrición, v.31, n.4, p.758, 1981.

MALLIPUDI, N.M.; STOUT, S.J.; DACUNHA, A.R.; LEE, A.H. Photolysis of imazapyr (AC 243997) herbicide in aqueous media. Journal of Agricultural and Food Chemistry, v.39, n.2, p.412-417, 1991.

MARTINS, D.; VELINI, E.D.; NEGRISOLI, E.; TOFOLI, G.R. Controle químico de Pistia stratiotes, Eichornia crassipes, e Salvinia molesta em caixas D'água. Planta Daninha, v.20, p.83-97, 2002.

(Edição Especial).

MARTINS, D.; CARBONARI, C.A.; TERRA, M.A.; MARCHI, S.R. Ação de adjuvantes na absorção e translocação de glyphosate em plantas de aguapé (Eichhornia crassipes). Planta Daninha, v.27, n.1, p.155163, 2009.

MONQUERO, P.A.; CHRISTOFFOLETI, P.J.; OSUNA, M.D.; DE PRADO, R.A. Absorção, translocação e metabolismo do glyphosate por plantas tolerantes e suscetíveis a este herbicida. Planta Daninha, v.22, n.3, p.445-451, 2004.

NEVES, T.; FOLONI, L.L.; PITELLI, R.A. Controle químico do aguapé (Eichhornia crassipes). Planta Daninha, v.20, p.89-97, 2002. (Edição Especial).

PESTER, T.A.; NISSEN, S.J.; WESTRA P. Absorption, translocation, and metabolism of imazamox in jointed goatgrass and feral rye. Weed Science, v.49, n.5, p.607612, 2001

RAJU, R.A.; REDDY, M.N. Control water hyacinth through herbicides. Industrial Farming, v.38, n.1, p.19-21, 1988.

RUBIM, M.A.L.; CAMARGO, A.F.M. Taxa de crescimento específico da macrófita aquática Salvinia molesta em um braço do rio Preto, Itanhaém, São Paulo. Acta Limnologica Brasiliensia, v.13, n.1, p.61-73, 2001. 
SATICHIVI, N.M.; WAX, L.M.; STOLLER, E.W.; BRISKIN, D.P. Absorption and translocation of glyphosate isopropylamine and trimethysulfonium salts in Abutilon theophrasti and Setaria faberi. Weed Science, v.48, n.6, p.675-679, 2000.

SHANER, D.L. Physiological effects of the imidazolinone herbicides, In: SHANER, D.L.; O'CONNER, S.L. (Ed.). The imidazolinone herbicides. Boca Raton, FL: CRC Press, 1991.

SILVA, M.A.S. Efeitos de períodos sem chuva, dosagens e volume de aplicação de glyphosate no controle de Brachiaria decumbens Stapf. 1996. 67p. Dissertação (Mestrado em Agronomia) - Universidade Estadual Paulista, Botucatu, 1996.

SOCIEDADE BRASILEIRA DA CIÊNCIA DAS PLANTAS DANINHAS . Procedimentos para instalação, avaliação e análise de experimentos com herbicidas. Londrina: SBCPD, 1995. 42p.
STEWARD, K.K. Nutrient removal potentials of various aquatic plants. Hyacinth Control Journal, v.8, n.2, p.34-35, 1970.

WEED SCIENCE SOCIETY OF AMERICA. Herbicide handbook. 8.ed. In: VENCILL, W.K. (Ed.). Lawrence, KS: WSSA, 2002. 493p.

WERLANG, R.C.; SILVA, A.A.; FERREIRA, L.R.; MIRANDA, G.V. Efeitos da chuva na eficiência de formulações e doses de glyphosate no controle de Brachiaria decumbens. Planta Daninha, v.21, n.1, p.121-130, 2003.

WERSAL, R.M.; MADSEN, J.D. Comparison of Imazapyr and Imazamox for Control of Parrotfeather (Myriophyllum aquaticum (Vell.) Verdc.). Journal of Aquatic Plant Management, v.45, n.2, p.132-136, 2007.

Recebido em $2 / 8 / 11$

Aceito em 6/3/13 